\title{
ADVANCED NURSING PRACTICE - EVOLUTION OR REVOLUTION?
}

\author{
Sandra Richardson, BA, Dip.Soc.Sci., Dip.Heal.Sci.(PG)
}

Nurse Researcher, Christchurch Hospital Emergency Department

\begin{abstract}
This article has been written to challenge the seemingly unquestioning acceptance of changing roles in New Zealand nursing. It is evident in nursing history that a number of issues, such as community versus hospital services and generalist versus specialist practice, tend to resurface in a cyclic manner. Characteristics that are currently associated with 'advanced' nursing roles can be recognised when the origins and development of nursing in New Zealand are traced. This gives rise to speculation that changes to nursing scope of practice are not so much revolutionary as evolutionary, and can perhaps be viewed as a reclaiming rather than a redefining of nursing roles.
\end{abstract}

Key Words: advanced practice, nursing boundaries, New Zealand nursing history.

\section{Introduction}

There is considerable interest in the area of 'advanced' practice roles for nurses, both nationally and internationally. A number of descriptors have been applied to these projected nursing roles often, in their multiplicity, more confusing than enlightening. The phrase 'advanced practice' tends to be used as an umbrella term encompassing many of the sometimes conflicting definitions associated with the concept. New Zealand (NZ) nursing appears to be embracing the re-conceptualisation of the scope and practice of nursing that these roles imply.

Historically nursing in New Zealand, as elsewhere, has been influenced by political, social and cultural factors as they emerge within its environment. This has resulted in a range of interpretation and varied implementations of the advanced nursing role (Denner, 1995; Scott, 1996; Sheehy \& McCarthy, 1998). Central to the concept of advanced practice is the notion of change that extends or expands existing boundaries, suggesting that innovation, revolution and reconstruction are part of this process. In contrast to this view, an analysis of nursing in early New Zealand suggests an alternative hypothesis namely that, rather than pushing against the boundaries of other professions, nursing is in fact reclaiming elements associated with earlier nursing roles.

Richardson, S. (2002). Advanced nursing practice - evolution or revolution? Nursing Praxis in New Zealand, 18(2), 33-41. 


\section{The problem of definition}

Within NZ interest in the advanced practice concept has been shown by individual nurses, professional nursing organisations, and at a political level (e.g. Ministry of Health, 1998; Jacobs, 1998; Litchfield, 1998; Nursing Council of New Zealand, 2000.). Yet, despite the considerable degree of interest, a central tension remains. There is no clear, generally accepted definition or understanding of the term 'advanced practice'. Instead several descriptions (Christensen, 1999; Litchfield, 1998; Ministry of Health, 1998; NENZ, 1998; Nursing Council of New Zealand, 1999, 2000) are being considered currently. The specifics of role description and implications in terms of professional boundaries continue to be debated. The development of advanced nursing practice has been linked with the concepts of enhanced professional standing and greater autonomy for nurses (O'Malley, Cummings \& King, 1996). Milstead (1997) goes further, asserting that the advanced nurse practitioner has an important political role, arguing that "if ANPs do not exercise the influence inherent in the role, professional nursing practice will revert to the dependent, handmaiden status of the early 20th century" (p.12). He goes on to identify the importance of ANPs becoming involved in shaping and setting public policy, operating at a national as well as a local level.

There is debate regarding the implications of adopting a wider scope of practice. In taking on a wider scope, other practice elements may be lost, altering the basic nature of nursing practice. Magennis, Slevin and Cunningham (1999) undertook a limited study of nurses' attitudes about expanded and extended nursing practices. While generally positive in their responses, the nurses surveyed nevertheless identified a perceived increase in vulnerability regarding the possibilities of exploitation, litigation and fragmentation of the basic nursing role.

Unlike such professions as medicine and law, the core elements and essential definition of nursing remains subject to debate. Henderson (1991) postulates that the need to define nursing will remain a perennial problem, “... because conditions change from one era to the next and change with the culture, or the nature, of a society" (p.6). This raises the question of why nursing is so difficult to define, and what effect failure to articulate its fundamental purpose and function has on the credibility of the claim to 'professionalism'. Nursing has been represented in a variety of ways throughout history, and the discourses that have arisen around these definitions remain influential today. The discourse of professionalism, for example, is evident in the rhetoric of nursing organisations and in the focus on developing such roles as the Nurse Practitioner (Nursing Council, 2000). Despite this emphasis, the influence of the 'nursing as vocation' discourse is still evident in references to nurses 'putting their patient first', and links with concepts of self-sacrifice and moral goodness as ascribed to the earlier Nightingale nurses. 
One debate concerning advanced nursing practice can be seen in terms of two competing discourses - those of nursing as an 'art' and as a 'science'. This debate can also be framed in terms of expanded versus extended practices, with the term 'extended' linked to the discourse of 'nursing as science', and that of 'expanded' to the discourse of 'nursing as art'. The extended role is described by Davis (1992) as one "... not included in basic nurse training, and comprises tasks normally undertaken by a doctor, but which may be delegated to a nurse who has received appropriate training and has been assessed competent" (p.37). Similarly, 'nursing as a science' involves a focus on the value of technical competence and skill level, drawing on the bio-medical model and such attributes as objectivity and efficiency. The expanded role can be defined as involving “... a more holistic approach to the development of the nurse's role: expanding their role around the needs of the patient, rather than simply taking on delegated medical tasks" (Davis, p.37). While this is a rather simplistic dichotomy, such generalisations indicate the wide spectrum of nursing practice.

In any study of history cycles are likely to be observed, and nursing history is no exception. It is commonly suggested that the mistakes of the past are being repeated, but it can also be argued that there is an opportunity to reclaim the successes of the past. There are a number of recurring issues specific to the nursing workforce, including shifts in focus regarding practice bases and the skills and knowledge needed to support these.

Within NZ, the issues associated with changing professional expectations are becoming increasingly evident in the reconfiguration of nursing aspirations and public perceptions. The movement towards 'advanced nursing practice' is one that can be seen as rooted in the historical development of nursing - NZ nurses have often shown innovation and flexibility in their roles and scope of practice. Nurses in the past have demonstrated responsiveness to the social mandate they hold, developing services that reflect the changing needs of society. The ability to accommodate such changes is evident in the emergence of uniquely $\mathrm{NZ}$ solutions such as the Nurse Maude District Nursing Service, Plunket and Karitane services. Debate about autonomy and independence in practice is often linked to advanced nursing practice, yet closer examination of the 'handmaidens' of the past working in such roles as 'back blocks' district nurses and native nurses shows that these women had considerable independence in their practice.

\section{Influences shaping NZ nursing}

Following the introduction of the welfare state, NZ has been viewed as a centre of innovation and forward thinking. The colonial heritage, while decried for its impact on the indigenous peoples of $\mathrm{NZ}$ (Cheyne, O’Brien \& Belgrave, 1997; Rata, 1986; Walker, 1986) also gave rise to a social framework within which a respect for the unconventional, and a valuing of 
initiative was evident (Cheyne, O'Brien \& Belgrave, 1997; Rattray, 1961). While, despite their enfranchisement, the role of women in society remained constrained, there were opportunities to move outside the traditional framework of western societies. The $\mathrm{NZ}$ nursing culture continued to be refined and re-focused as a result of influences from colonialism, social experimentation and, more recently, the gradual acknowledgement of the values and aspirations of the Tangata Whenua (indigenous people).

The first of the 'Nightingale' nurses arrived in NZ in 1883, bringing with them the values and morals, as well as the clinical and theoretical knowledge, that formed the basis of their training (Burgess, 1984; Rodgers, 1985). These nurses transposed the structure and purpose of nursing as it had developed in the United Kingdom, and the perceived efficiency and effectiveness of their practice soon saw this emulated in the colonies. There were few formally trained nurses in $\mathrm{NZ}$ prior to this period, although nursing actions were evident as described in the histories and biographies of this period (Rodgers, 1985; Rattray, 1961).

Nightingale is seen as a seminal influence on nursing practice, and the widely acknowledged founder of modern nursing. She determined the structure and ethos of Western secular nursing practice and education, significantly influencing public health beliefs and introducing the concept of the 'trained' nurse (Selanders, 1998). Prior to Nightingale's involvement, nursing was a disreputable, menial occupation, poorly paid and poorly respected. Commonly recognised caricatures such as that of 'Sairey Gamp' portrayed by Dickens were accepted as accurate representations of the contemporary state of nursing. Nightingale's description of nursing (cited in Bowman, 1967, p.19) was that it was undertaken by those "too old, too weak, too drunken, too stolid or too bad to do anything else". In $\mathrm{NZ}$, early state provided nursing care was not dissimilar, with hospital staff consisting of a Master and Matron together with any additional help that could be found. These untrained personnel were not held in high regard, and hospitals were seen as a place of last resort, a refuge for the poor and indigent. Lambie (1947) describes the type of women employed as "uneducated, rough and often uncouth in their conduct and this was reflected in the state of the wards and the care of the patients" ( p.3). It was not until the late 1870s that the first regulations regarding nursing standards were introduced. Requirements for nurses working at Christchurch Hospital were identified as follows: "Nurses must be able to read and write, they must treat the sick with kindness, see that all patients are in bed after the doors are locked and put the gas down on retiring to rest" (Buchanan, 1951, p.69).

Within NZ opportunity to stretch and even breech some of the traditional boundaries constraining women in society was apparent, and influenced the way in which nursing developed. This is illustrated in the work of Grace Neill, trained under the Nightingale system, who became the first female 
assistant Inspector for the Department of Hospitals, Asylums and Charitable Aid (Neill, 1961). Her duties included inspecting the various hospitals, during which she became aware of a considerable disparity in nursing standards. This, together with her interest and involvement in the activities of the International Council of Nurses, led to her supporting the establishment of a State Examination for Nurses. In $1901 \mathrm{NZ}$ passed the first registration act for nurses in the world. This specified a course of three years duration, culminating with the State Examination and entry to the Register for successful participants. Neill expanded the interests of the state, identifying the role of central coordination and regulation in regard to the provision of nursing related services. The place of the political, as well as the professional, agenda in shaping the role of the nurse was becoming formally vested in the functioning of the state.

The introduction of registration for nurses was seen by many as providing a professional base, but this conceptual shift regarding the role and place of nursing in society was not universally accepted. Sargison (1993) suggests "some doctors were wary of 'overtrained' nurses who might threaten their prestige, and some parliamentarians feared professional examinations might undermine the 'womanly instinct' for nursing" (p.19). The concept of overtrained nurses is still evident in contemporary society. The fear of 'advanced' nursing roles resulting in some form of mutant mini doctor has been well articulated in the nursing literature (Shepherd, 1993; Denner, 1995; Mitchinson \& Goodlad, 1996). There is a tendency to question the relevance of some aspects of nursing knowledge and skills. Rather than questioning the appropriateness of specific elements of nursing also common to other professions, an acknowledgement of the influence of the conceptual and practice frameworks within which these occur highlights their significance when part of nursing practice. As the nursing profession focuses on the development and recognition of professional attributes, the value of specific nursing knowledge and the unique nature of the nurse patient interaction is emphasised. The public and members of the health care team need an understanding of the distinctive nursing contribution. Equally, nurses themselves need to clearly articulate what it is about their practice that is unique and valuable.

The expected range of duties and work conditions associated with early nursing positions required a degree of flexibility and adaptability. Ellen Dougherty became the world's first Registered Nurse. She studied chemistry at Wellington Public Hospital so that "when she was appointed Matron of the Palmerston North Hospital she was well equipped for her own responsibilities, and did her own dispensing. In an emergency she could operate, amputate and set broken limbs" (Rattray, 1961 p.30). Conditions in the colony remained primitive for some time, with nursing staff having to deal with a range of environmental hazards and limitations. One English trained nurse, Miss Blackley, arrived for work 
at New Plymouth Hospital in 1886, where she found

there was neither bathroom, wash house nor mortuary. Everything had to be carried to the wards by way of the kitchen or front entrance. There was an inadequate supply of running water. Operations were performed at night by candlelight in the men's lavatory, where the only tap was installed (Rattray, 1961, p.30).

The value placed on willingness, flexibility and initiative continued during the early 1900s, and differences between the new breed of colonial nurses and those from the 'home countries' were becoming increasingly evident. The following comments were written in response to a British Journal of Nursing editorial, which outlined some of the concerns and shortcomings identified with nursing work in the colonies. The British Journal noted that "life in a well-appointed nurse' home with its comfort, clockwork regularity and sufficiency of service is not good preparation for roughing it in the colonies" (anon. 1912, p.93). In response to this comment, the New Zealand Nursing Journal notes "it has certainly been found that some English nurses working in the colonies appear to expect a great deal of waiting upon ... colonial nurses at Home have been commended for their readiness to put their hands to anything" (anon. 1912, p.94.)

\section{Specialist or Generalist?}

Specialisation in nursing practice is not a new concept. Nightingale herself noted that "a nurse should do nothing but nurse. If you want a charwoman, have one. Nursing is a speciality" (Skretkowicz, 1992, p.65). Early movements aimed at providing advanced education for nurses in NZ were also structured along specialty lines, with courses initially designed to provide for nurses working in public health, administration and education (Lambie, 1947; Burgess, 1984).

An alternating focus on community versus hospital based care can also be seen in nursing history. This is linked not only to government policies but also to changing social expectations and technological developments. The link between nursing and hospital based care developed following the introduction of the Nightingale training system. This system of education was based on the apprenticeship model. Nursing students were to provide care within the hospital for minimal recompense, but also be given time for study and to receive instruction. In practice, the amount of time allocated for theoretical education was quickly reduced, with the nursing students proving a valuable commodity, and one that soon became essential to the economic viability of the hospital system.

Following on from this early association, nursing became strongly linked to hospital based care, in both a practical and ideological sense. Practical links included the greater reliance on nursing actions following the introduction of Listerian theories of asepsis, which necessarily involved greater collaboration between doctors and nurses. The practice links were soon followed by ideological ones, with medicine seen as the role model for professional attainment. Nursing was increasingly described in terms of 
'agency' for the performance of medical tasks, and nursing rhetoric took on the vocabulary and focus of the bio-medical model. The 'handmaiden' image was thus reinforced, and the relationship between nursing and medicine increasingly formalised, with nurses identified in a dependent role. As the practice of medicine became increasingly specialised, so too did nursing, with a shift away from the generalist approach to one of specific expertise. Ideologically, as nurses moved to a more subservient role, oppressed group behaviour became increasingly evident, including the desire to emulate the socially valued professional model.

Within NZ, the shift back to a community focus is again apparent, and the need for nurses with a sound generalist base has again emerged. There is growing pressure for nurses working in isolated and often rural areas, to expand their practice. The Ministerial Taskforce on Nursing (1998) identified the potential role of nurses in improving access to health care services. Taken together with the growing difficulty of attracting General Practitioners to rural areas, and the current moves towards nurse prescribing, it seems likely that advanced practice roles may be seen as the solution to both physician shortages and nurses' professional aspirations.

Nurses in colonial NZ initially developed as generalists, with a degree of independence despite the lack of formally vested autonomy. Nurses were valued within the communities where they worked, and were expected to bring a range of skills and knowledge to their practice. This practice has changed over time, influenced by changes in medicine and health policy, and the movement toward specialisation, largely occurring in the hospital setting. In linking nursing to the hospital system, it was inevitable that the relationship between nursing and medical staff has become more stratified, with an underlying rigidity that is only now being challenged. Shifts in educational policies have also mirrored this movement. As nurses first undertook formal education, the link was clearly to medicine, hospitals, and specialty practice. It may be suggested that the development of the comprehensive nursing programs, offered through polytechnics, began to modify this focus, by moving back toward a single, integrated registration producing a generalist practitioner. The on-going focus of post-graduate courses appears to be to return to a specialty focus. However, based on the pattern of swings that is evident in a review of the past, I would suggest that the advanced practitioner of tomorrow will continue the cycle, returning to a comprehensive general expertise, developing skills and attributes that are readily transferable within a range of advanced settings. While there will still be specialists within advanced nursing practice, their strength will be in critical thinking, the ability to see the possibilities in a situation and envisage a range of outcomes, and with the ability to justify their practice.

While colonial nurses were not able to act autonomously in the sense that we use the term today, they were required often to work independently, to make their own decisions, and to determine the appropriate interventions for the 
situation. It may be that as nursing became more closely linked to medicine, that the options for nursing to expand became more limited, and that nurses in fact saw the extension of their role as the way forward, in a society that valued the scientific, measurable and task based role. The recognition of nursing as a separate and legitimate domain, and the growing base of nursing epistemology and methodology, have opened the way for nurses to again expand as well as extend the scope of their practice.

\section{Conclusion}

The NZ nursing profession, like those in many other countries, is showing interest in the concept of advanced nursing practice. There is considerable debate about this movement, including issues of definition, scope of practice and the need for credentialling. A number of attributes and attitudes are generally associated with practice at an advanced level, many of which can be recognised in a review of $\mathrm{NZ}$ nursing history. As the nursing profession moves forward, it is important not to lose sight of the past - the potential lessons are not only those found in the story of oppression and medical domination. Nurses in $\mathrm{NZ}$ have demonstrated innovation and advancement in practice, a heritage to be acknowledged and respected. The current movement towards advanced nursing practice is a continuation of this legacy, although whether it will be recognised as an inevitable evolution or a radical revolution remains to be seen.

\section{References}

Anonymous. (1912). State Registration. Kai Tiaki 5(4), 127.

Bowman, G. (1967). The lamp and the book. London: The Queen Anne Press Ltd.

Buchanan, D. I. (1951). Presidential address. The New Zealand Nursing Journal, 44(1), 68-79.

Burgess, M. (1984). Nursing in New Zealand society. Auckland: Longman Paul Ltd.

Cheyne, C., O’Brien, M., \& Belgrave, M. (1997). Social policy in Aotearoa New Zealand: A critical introduction. Auckland: Oxford University Press.

Christensen, J. (1999). Integrating the terminology and titles of nursing practice roles: Quality, particularity and levelling. Nursing Praxis in New Zealand, 14(1), 4-11.

Davis, P. (1992). Health and health care in New Zealand. Auckland: Longman Paul Ltd.

Denner, S. (1995). Extending professional practice: Benefits and pitfalls. Nursing Times, 91(14), 27-29.

Henderson, V. (1991). The nature of nursing. New York: National League for Nursing press.

Jacobs, S. (1998). Advanced nursing practice in New Zealand: 1998. Nursing Praxis in New Zealand, 13(3), 4-12. 
Lambie, M. (1947). Historical development of nursing in New Zealand 1840-1946. Wellington: Department of Health.

Litchfield, M. (1998). The scope of advanced nursing practice. Nursing Praxis in New Zealand, 13(3), 13-24.

Magennis, C., Slevin, E., \& Cunningham, J. (1999). Nurses' attitudes to the extension and expansion of their clinical roles. Nursing Standard, 13(51), 32-36.

Milstead, J. A. (1997). Using advanced practice to shape public policy: Agenda setting. Nursing Administration Quarterly, 21(4), 12-18.

Ministry of Health. (1998). Report of the ministerial taskforce on nursing: Releasing the potential of nursing. Wellington: Author.

Neill, J. O. C. (1961). Grace Neill: The story of a noble woman. Christchurch: N.M. Peryer Ltd.

Nurse Executives of New Zealand. (1998). Developing and supporting advanced practice roles. Auckland: Author.

Nursing Council of New Zealand. (1999). Framework for post registration nursing education. Wellington: Author.

Nursing Council of New Zealand. (2000). Regulation of the nurse practitioner (draft discussion document). Wellington: Author.

O’Malley, J., Cummings, S., \& King, C. (1996). The politics of advanced practice. Nursing Administration Quarterly, 20(3), 62-72.

Rata, M. (1986). Opening address. Nursing educational hui: Ratana Pa. Wanganui: National Council of Maori Nurses.

Rattray, J. (1961). Great days in New Zealand nursing. Wellington: A.H. \& A.W. Reed.

Rodgers, J. (1985). Nightingale's contribution to New Zealand nursing. Horizons of care conference, Norman Peryer Forum. Wellington: NERF.

Sargison, P. (1993). Notable women in New Zealand health. Auckland: Longman Paul Ltd.

Scott, H. (1996). Unwanted medicine: The delegation of doctors' roles (editorial). British Journal of Nursing, 5(17), 1028.

Selanders, L. C. (1998). Florence Nightingale: The evolution and social impact of feminist values in nursing. Journal of Holistic Nursing, 16(2), 227-243.

Sheehy, C. M. \& McCarthy, M. C. (1998). Advanced practice nursing: Emphasising common roles. Philadelphia: F. A. Davis Co. Ltd.

Shepherd, J. (1993). Nurses are changing not extending their roles (editorial). British Journal of Nursing, 2(9), 447.

Skretkowicz, V. (1992). Florence Nightingale's notes on nursing. London: Scutari Press.

Walker, R. (1986). The meaning of biculturalism. Nursing educational hui, Ratana Pa. Wanganui: National Council of Maori Nurses. 